\title{
Assessing the Potential Impact of General Collective Intelligence
}

\author{
Andy E. Williams, Nobeah Foundation, Nairobi, Kenya
}

\section{Keywords:}

General Collective Intelligence, Collective Intelligence, general problem solving ability, c factor

\begin{abstract}
:
The concept of General Collective Intelligence or GCI is summarized, and the potential for GCI to exponentially increase the general problem-solving ability of the group so that it is far larger than that of any individual in the group, and therefore the potential for GCI to exponentially increase the ability of groups to impact collective outcomes are explored. GCI is represented as a repeating pattern, beginning with a first order GCI, then progressing to an Nth order one, where N might be limited by the resources available, and where each order is suggested to create the potential for an exponential increase in general problem-solving ability. Finally, the claim that such an exponential increase in potential for impact on any general problem makes GCI the most important innovation in human history, and the most important innovation in the near term future, until the transition to second order GCI, is explored.
\end{abstract}

\section{What is General Collective Intelligence?}

General Collective Intelligence or GCI has been defined as the general intelligence factor characteristic of groups. Woolley (2010). GCI has also been defined as any software platform organizing groups into a single collective intelligence with a group general intelligence factor having the potential to be significantly higher than the general intelligence factor of any individual in the group, thereby having the potential for general problem-solving ability vastly greater than that of any individual in the group as in Williams (2019a), and Williams (2020a). From this perspective, since the only system with human-like general problem-solving ability or greater is the human cognitive system, a GCI must define an analogous concept of collective cognition for the group, as well as a mechanism by which that collective cognition might not only be achieved, but might be vastly increased. Researchers at the MIT Center for Collective Intelligence (CCI) have associated the concept of an extremely high group general intelligence factor with that of a supermind as in Amdt (2020). Similar to the $\mathrm{g}$ factor (g) for general individual intelligence, some collective intelligence researchers have attempted to extract this general collective intelligence factor (c factor) for groups as an indication of a group's ability to perform a wide range of tasks. Just as $g$ is highly interrelated with the concept of IQ as in Jensen (1992), and Jensen (1998), as a measurement of collective intelligence this c factor has been interpreted as intelligence quotient for groups (Group-IQ).

The distinction between a Collective Intelligence (CI) solution and a GCI solution has been suggested in Williams (2019a) to be that analogous to the difference between "narrow AI" and Artificial General Intelligence or AGI as in Bundy (2017), in that CI targets a specific problem or range of problems, and therefore constrains a group to do so. Groups not constrained to solve a specific problem by a CI solution might have some degree of general problem solving ability and therefore might have some $\mathrm{c}$ factor. But groups constrained to solving a specific problem must by definition lack general problemsolving ability, and therefore must lack a c factor. Where a CI might use the intelligence of crowds to improve outcomes of decision-making by choosing a more optimal solution, GCI is intended to create the capacity to improve outcomes of decision-making much further through enabling the group to address any general problem, including the choice of which problem to solve. In other words, the c factor of CI solutions is limited to the degree that such solutions lack the capacity to target any general 
problem. Some specific limitations intended to be addressed by GCI solutions, that are suggested in Williams (2019a) to be characteristic of CI solutions, include the fact that in a CI there are barriers to participation in group decision-making. Individuals might not be able to join the group, and might not be eligible to provide input on certain decisions or on the way decisions are made. And where individuals are able to provide such input, the methods for evaluating suggestions are decided by the designers of the CI and so are free to be aligned with the cognitive biases, self-interests, and other predispositions of those designers. So that even where an individual might make a suggestion, the process by which those suggestions are evaluated might not function to choose the optimal suggestion in terms of functioning to achieve the outcome targeted by the CI designer, rather than the optimal collective outcome for the group as in Williams (2019a) From this perspective, in a complete implementation of GCI, or equivalently, in a CI initiative with a sufficiently high GCI or c factor, it would be expected that each individual must be able to suggest an improvement to any concept the group is working on, including the design of the CI itself, and the CI must create the capacity to consider all those suggestions in a way that converges on the optimal collective choice.

By the same reasoning, a CI initiative with a high GCI factor might be expected to have a high ability to solve any general problem, such as the problem of forming collaboration. By contrast the lack of sufficient GCI to define decentralized processes by which collaborations can be formed, and by which those actors can influence the agenda of group efforts, leaves the agenda to become aligned with centralized interests, namely those of the initiative designers, potentially excluding the input of those the initiative is supposed to serve. This has been suggested to be a critical flaw with group decisionmaking in the absence of GCI. Resulting in perverse incentives that have been suggested to prevent global collective impact such as the sustainable development goals from being reliably achievable without GCI as in Williams (2020b). Many CI institutes and initiatives can be criticized as lacking GCI because they lack any public outreach mechanism by which collaborations may reliably be formed or by which feedback might be provided. From this perspective, a key test of whether a CI initiative has high collective intelligence (a high GCI factor) might then be the degree to which any random individuals or initiatives are able to form a cooperation with that CI initiative. A high GCI factor (c factor), that is a high ability to solve any general problem, would be expected to create the potential to find a mechanism for cooperation in all cases. The existence of general problem-solving ability and therefore the validity of this $\mathrm{c}$ factor might not provable without a validated model of collective cognition to provide a concrete definition of that c-factor as distinguished from the intelligence of any individual in the group. But if the ability to solve a sufficient number of specific problems can be demonstrated, in a broad enough range of cases, the probability that general problem-solving ability exists might be statistically assumed. Successfully demonstrating the capacity to form cooperation with other individuals and initiatives in a large enough range of cases from this perspective is potentially one demonstration of a high GCI factor, though the distinction between the problem-solving ability of an individual in the group as opposed to the problem-solving ability characteristic of the group would need to be made in order to confirm this. However, the failure to do so is potentially a demonstration of a lack of GCI, though again the distinction between a lack of problem-solving ability arising from a poor system of GCI, and a lack of problem-solving ability arising from other factors such as small group size would need to be made in order to confirm. 
One central challenge for GCI is solving the problem that groups might collectively lack the capacity to even recognize the opportunity to make truly revolutionary impact, which GCI has been proposed to be as in Williams (2020c). A complex concept that has revolutionary implications in every field of science can often be simplified to the point that it can be reliably understood. But no reliable mechanism for sharing simplifying generalizations between the scientists or other thought leaders currently exists. Instead there are a growing number of scientific journals in each field that require a high degree of specialization. For a theory to be acknowledged as having profoundly transformative impact that crosses a wide range of different disciplines, experts in those disciplines will have to agree. But as the number of such experts that needs to be convinced rises, at some point it is outside the capacity of any individual human intelligence to communicate to all the people in all those different disciplines at a sufficient level of specificity and expertise to be published in those journals and so to convince those experts. In other words, when ideas are sufficiently revolutionary, groups lack the collective intelligence to reliably recognize them as such.

\section{Partial Literature Review Regarding General Collective Intelligence}

Theories have been developed regarding the mechanisms by which General Collective Intelligence might operate as in Krafft (2019), and methodologies by which a supermind (presumably a GCI) might be implemented have been explored as in Amdt (2020), and in addition a number of other papers refer to the concept of General Collective Intelligence. However, neither those theories of General Collective Intelligence, nor that concept of a supermind define collective cognition, appear to define any specific mechanism by which a significant increase in general problem-solving ability can be achieved. This claim was examined through a partial review of the academic literature containing the term "general collective intelligence" in the Google scholar website. Of the one hundred and sixty-five results returned, the first seven of a total of fifteen pages were reviewed. In addition, some more specific searches were used to find additional results from the remaining pages, such as "general collective intelligence" and algorithm to find additional papers describing GCI implementations. Also, additional searches using the search terms "general collective intelligence" and supermind, or "supermind" alone, were used to find additional papers describing GCI with the potential for a greatly increased GCI factor (c factor). After removing dissertations that have not been published as papers, all the entries were found to conform to the five categories below:

\section{Category (Number) Description}

Mentions GCI Concept Mentions or provides reference to work that mentions the potential existence of a GCI factor (c factor) without describing a model of implementation, or work that refutes the existence of such a factor: Woolley et al (2010), Woodley and Bell (2010), Woolley et al (2015), Engel et al (2015), Barlow and Dennis (2016), Credé and Howardson (2017), Kim et al (2015), Nehra et al (2020), Gimpel et al (2018), Joseph et al (2019), Barlow and Dennis (2017), Bernstein et al (2011), Mayo and Woolley (2019), Martin-Raugh et al (2020), Cushman and Gershman (2019)

Mentions GCI Implementation (12) Describes or provides reference to material describing a model for an implementation of GCI (a system of organization with general problem- 
solving ability), or for a system believed to have a GCI factor (c factor) or for the mechanism by which a GCI factor might be achieved in groups: Krafft (2019), Reia et al (2019), Dai et al (2020), Palak and Nguyen (2017), Krafft (2017), Toret and Calleja (2014), Kudyba et al (2020), Morano et al (2018), Callaghan (2015), Sotiriadis (2010), Pease et al (2020), Woolley and Hashmi (2013)

Mentions GCI with Describes or provides reference to material describing a methodology or Greatly Increased GCI general concept for implementing a greatly increased GCI, or explicitly Factor (Supermind) without Model for Cognitive Ability to Justify Claim (3) describing a supermind (a system of organization that is suggested to give a group greatly enhanced general problem-solving ability). A precise methodology for defining intelligence and for confirming the potential capacity of a supermind for increasing intelligence is not provided: Callaghan (2016), Malone (2020), Malone (2018), Steinhart (2003), Laubacher (2020), Metcalf (2019), Bringsjord (2003)

Mentions GCI with Describes or provides reference to material describing an implementation of Greatly Increased GCI a GCI, the problems it solves, a definition of individual or group intelligence, (Supermind) with Model as well as a first principle analysis of the increase in general problem-solving for Cognitive Ability to Justify Claim (11) entailed by the concept of a "supermind", and the exact GCI functions required to achieve it. The main papers outlining this model are Williams (2020a), Williams (2020b), multiple other papers by the same author exploring implications of the model in other areas, also in 2020, were skipped.

False Positives (7) Papers containing text strings that use the term "general collective intelligence" in a context that does not refer to a GCI factor (c factor) such as "in general, collective intelligence can be assumed to be ...". Or works of fiction. References are not provided for these papers.

As an example of the category "Mentions GCI Concept" is Woolley et al (2010) which mentions "converging evidence of a general collective intelligence factor that explains a group's performance on a wide variety of tasks". Examples of the category "Mentions GCI Implementation" are Krafft (2019) which describes the mechanism by which a general collective intelligence factor might operate, or Palak and Nguyen (2017) which describe how prediction markets might demonstrate a general collective intelligence factor. As examples of the category "Mentions Supermind" is Malone (2018) which popularized the concept of a supermind in the CI community, and Steinhart (2003) which explored the concept in the abstract as applicable to any entity, as well as Laubacher (2020) which explored the application of the concept in the specific case of creating the capacity to implement a supermind.

In the category "Mentions GCI or Supermind with Model for Cognitive Ability to Justify Claim" only one model for General Collective Intelligence or GCI that proposes to define the complete set of functionality required by a GCI has been developed as described in Williams (2020a). For this reason, and because this model is new, the research exploring an actual model for GCI with the potential for a vastly increased general collective intelligence factor, and exploring the implications of this model in healthcare, design, manufacturing, and a wide variety of other areas are solely from this single author, Andy E. Williams.

\section{Unique Properties of the Model of General Collective Intelligence}

This model is claimed to have the potential to organize groups into a single collective intelligence with the potential for general problem-solving ability that is not only significantly greater than that of 
anyone in the group, but exponentially greater. This model of GCI provides an objectively defined model of the GCI or c factor as the volume of the collective cognitive space that can be navigated per unit time, so that an exponential increase in the $\mathrm{c}$ factor is well-defined as in Williams (2020d). While computers can solve problems exponentially more quickly than human beings might, there is currently no known system with the ability to execute general problem solving ability at exponentially greater speed and scale, in order to exponentially increase this c factor for the group. In the proposed model of GCI this exponential increase is achieved through defining functional models of concepts, which when combined with this model of cognition to provide an interpretation of meaning, are suggested here to equate to semantic models. These models allow simplifying generalizations of any problems or solutions to potentially be exchanged at orders of magnitude greater speed and scale. In this functional model, as ability to exchange simplifying generalizations rises to a well-defined point, this increase results in the potential for an exponential increase in general problem-solving ability for the group as in Williams (2020d). This potential for greater problem-solving ability using these models is achieved through a set of GCI functionality designed to remove the barriers against reliably converging on solutions that optimize any group outcomes targeted, and removing the barriers against doing so at that increased speed and scale.

GCI is in essence collective cognition. Any collective reasoning process the collective cognition has learned might potentially be executed to navigate the collective conceptual space from a set of concepts representing a collective problem, to a set of concepts representing its solution. Just as an individual human cognition might execute any reasoning process it has learned. In this way a GCI has the potential to incorporate any algorithm automating any process of cooperation where doing so is projected to achieve optimal collective outcomes. That is, it has the potential to incorporate any specific CI solution, or any functionality of any GCI solution, in order to optimize outcomes in any given context.

GCI also incorporates both AI and AGI to create intelligent agents that represent each individual in interacting with other individuals, so that it is possible for individuals to interact at exponentially greater speed and scale as in Williams (2020b). In comparison to speeds at which groups might execute processes manually, this enables a GCI to instantaneously form communities to achieve impact on virtually any purpose as in Williams (2020b).

Defining problem-solving ability in terms of ability to impact an outcome, an exponential increase in problem-solving ability over that of any individual also implies an exponential increase in capacity to impact the external world, one measure of importance as in Williams (2020d). This exponential increase in problem-solving has been suggested to be a repeating pattern with a GCI consisting of GCI's (a second order GCI) having the potential for exponentially greater general problem solving ability than a GCI (i.e. a first order GCI). Because of this potentially exponential increase in impact, this transition from individual human intelligence to GCI has been suggested to be the most important innovation in human history, and the most important innovation in the immediate future until the transition from GCI to a second order GCI creates another exponential increase in capacity for impact as in Williams (2020c).

In design as in Williams (2020e), manufacturing as in Williams (2020f) and Williams (2020g), healthcare as in Williams (2020h), physics, mathematics, and a wide range of other subjects, the self- 
assembly in terms of functional components by group members that is characteristic of GCI has been suggested to have the capacity to reliably create concepts far beyond the capabilities of individuals or any current non-GCI orchestrated groups as in Williams (2020i). The reasoning behind this claim is that just like a target can't reliably be hit when it's too far away for the shooter to determine whether it's one object, two objects, or a thousand separate objects, a problem that's outside of the individual or group's ability to clearly resolve is one that cannot be reliably solved. And solutions that are too complex to be within the group's ability to clearly resolve, are solutions outside the ability of the group to discover. One solution to the problem of the target being outside of the shooter's ability to resolve is to create intelligent bullets. Bullets that travel until the target is within a range at which it can be resolved clearly, and that then decide their own target far more precisely than the shooter could achieve. In the same way, by decomposing problems into functional components that individuals might achieve, this model of GCI is intended to enable groups to self-assemble into solutions that might not have been anticipated by any individual in the group as in Williams (2020e).

This model of GCI is part of a more general model of cognition that has also been used to define what has been suggested to be the first model for Artificial General Intelligence (AGI) with the potential for human-like general problem-solving ability as in Williams (2020j). In this model of cognition, an exponential increase in intelligence has been suggested to be a repeating pattern of transition to higher orders of organization. These models arise from the common approach of Human-Centric Functional Modeling (HCFM) as in Williams (2020k). In facilitating a Functional Modeling Framework (FMF) as in Williams (20201) defining simple functional models that attempt to represent all the functions of cognition and of each other part of the human system, HCFM enables behaviors of these complex systems to be reliably understood. HCFM attempts to formalize the methodology by which Eastern existential philosophies such as yoga rely on the use of simple cognitive models to reliably gain the capacity to navigate the cognitive system to an understanding of complex phenomena in the world around the practitioner, where that understanding is proved to be true in that it reliably functions to achieve a specific outcome such as well-being as in Williams (2020m).

\section{Conclusions}

The claim that any single innovation might be the most important in human history might be impossible to come to consensus on from a subjective standpoint. However, from a functional standpoint, defining importance in terms of impact, any innovation with the potential to drive an exponential increase in the impact that has been historically achievable is clearly more important than any other innovation in the past. And while an AGI might also be able to achieve such an increase in impact, that impact could not be reliably aligned with mankind's collective interests. On the other hand, a GCI, as defined in this paper, is by definition aligned with collective interests. While such an AGI might certainly be an important threat if it's interests conflicted with mankind's own, the term "importance" is more commonly associated with that which creates opportunities to achieve beneficial outcomes. If this model of GCI does in fact have the potential to exponentially increase beneficial impact on any general problem over what is possible for any individual or group without GCI, from this perspective it would clearly be the most important innovation in human history, as well as in the immediate future until the transition to a second order GCI.

\section{References}


Woolley, Anita Williams; Chabris, Christopher F.; Pentland, Alex; Hashmi, Nada; Malone, Thomas W. (29 October 2010). "Evidence for a Collective Intelligence Factor in the Performance of Human Groups". Science. 330 (6004): 686-688. Bibcode:2010Sci...330..686W. doi:10.1126/science.1193147. PMID 20929725. S2CID 74579.

Williams, Andy E. (2019), "The Relationship Between Collective Intelligence and One Model of General Collective Intelligence", Computational Collective Intelligence, Cham: Springer International Publishing, pp. 589-600, ISBN 978-3-030-28376-6, retrieved 2020-08-17

Williams, Andy E. (2020a) Williams, Andy E (2020-04-30). "A Model for General Collective Intelligence". doi:10.31730/osf.io/6u984.

Arndt, Felix (2020-05-27). "Book Review: Superminds: The surprising power of people and computers thinking together". Organization Studies: 017084062092998. doi:10.1177/0170840620929989.

ISSN 0170-8406.

Jensen, Arthur R. (September 1992). "Understanding in terms of information processing". Educational Psychology Review. 4 (3): 271-308. doi:10.1007/BF01417874. ISSN 1040-726X.

Jensen, Arthur R. (1998). The g factor : the science of mental ability. Praeger Publishers. ISBN 0-27596103-6. OCLC 470276000.

Bundy, Alan (2017-05). "Preparing for the future of Artificial Intelligence". AI \& SOCIETY. 32 (2): 285-287. doi:10.1007/s00146-016-0685-0. ISSN 0951-5666. Check date values in: |date= (help)

Williams, Andy E (2020-04-16). "General Collective Intelligence and the Constraints to Group Decision-Making". doi:10.31234/osf.io/6gten.

Williams, Andy E (2020-04-20). "The Case for General Collective Intelligence Rather than Artificial General Intelligence being the Most Important Human Innovation in the History and Future of Mankind". doi:10.31730/osf.io/z8b7r.

Krafft, Peter M. (April 2019). "A Simple Computational Theory of General Collective Intelligence". Topics in Cognitive Science. 11 (2): 374-392. doi:10.1111/tops.12341. ISSN 1756-8757.

Williams, Andy E (2020-07-11). "Human Intelligence and General Collective Intelligence as Phase Changes in Animal Intelligence". doi:10.31234/osf.io/dr8qn.

Williams, Andy E (2020-08-18). "General Collective Intelligence, Human-Centric Functional Modeling and the Current Limitations on Collective Design Cognition". doi:10.31730/osf.io/eh3u8.

Williams, Andy E (2020-08-17). "General Collective Intelligence as Biomimicry of Nature's Design and Manufacturing Processes". doi:10.31730/osf.io/92rqx. 
Williams, Andy E (2020-08-17). "The Necessity of General Collective Intelligence Driven Processes in Achieving Pervasive Manufacturing". doi:10.31730/osf.io/7n258.

Williams, Andy E (2020-04-30). "The Collective Intelligence based Program to Accelerate Achievement of the Sustainable Development Goals as a Case Study for Collectively Intelligent Program Design". doi:10.31235/osf.io/r2dxq.

Williams, Andy E (2020-04-16). "General Collective Intelligence and the Constraints to Group Decision-Making". doi:10.31234/osf.io/6gten.

Williams, Andy E (2020-04-20). "A Model for Artificial General Intelligence". doi:10.31730/osf.io/xtq2v. As pre-print. Also in print in the proceedings of AGI 20.

Williams, Andy E (2020-05-15). "Use of Human-Centric Functional Modeling to Maximize Convergence in Integrative Research". doi:10.31730/osf.io/jv6h8.

Williams, Andy E (2020-04-16). "A Human-Centric Functional Modeling Framework for Defining and Comparing Models of Consciousness and Cognition". doi:10.31234/osf.io/94gw3.

Williams, Andy E (2020-04-27). "A Mathematical Model for Identifying Truth in Observations Made within Individual Human Self-Awareness". doi:10.31730/osf.io/4nsgk.

Michael A. Woodley, Edward Bell, Is collective intelligence (mostly) the General Factor of Personality? A comment on Woolley, Chabris, Pentland, Hashmi and Malone (2010), Intelligence, Volume 39, Issues 2-3, 2011, Pages 79-81, ISSN 0160-2896, https://doi.org/10.1016/j.intell.2011.01.004.

Woolley, A. W., Aggarwal, I., \& Malone, T. W. (2015). Collective Intelligence and Group Performance. Current Directions in Psychological Science, 24(6), 420-424.

https://doi.org/10.1177/0963721415599543

Measuring Collective Intelligence in Groups: A Reply to Credé and Howardson, Anita Williams Woolley, Yeonjeong Kimb, Thomas W. Malone, working paper (2018)

David Engel, Anita Williams Woolley, Ishani Aggarwal, Christopher F. Chabris, Masamichi Takahashi, Keiichi Nemoto, Carolin Kaiser, Young Ji Kim, and Thomas W. Malone. 2015. Collective Intelligence in Computer-Mediated Collaboration Emerges in Different Contexts and Cultures. In Proceedings of the 33rd Annual ACM Conference on Human Factors in Computing Systems (CHI '15). Association for Computing Machinery, New York, NY, USA, 3769-3778.

DOI:https://doi.org/10.1145/2702123.2702259

Jordan B. Barlow \& Alan R. Dennis (2016) Not As Smart As We Think: A Study of Collective Intelligence in Virtual Groups, Journal of Management Information Systems, 33:3, 684-712, DOI: $10.1080 / 07421222.2016 .1243944$ 
Credé, M., \& Howardson, G. (2017). The structure of group task performance-A second look at "collective intelligence": Comment on Woolley et al. (2010). Journal of Applied Psychology, 102(10), 1483-1492. https://doi.org/10.1037/ap10000176

Sandro M. Reia, André C. Amado, José F. Fontanari, Agent-based models of collective intelligence, Physics of Life Reviews, Volume 31, 2019, Pages 320-331, ISSN 1571-0645, https://doi.org/10.1016/j.plrev.2018.10.004.

Kim YJ, Engel D, Williams Woolley A, Lin J, McArthur N, Malone TW. Work together, play smart: Collective intelligence in League of Legends teams. Collective Intelligence Conference. 2015.

Woolley A.W., Hashmi N. (2013) Cultivating Collective Intelligence in Online Groups. In: Michelucci P. (eds) Handbook of Human Computation. Springer, New York, NY. https://doi.org/10.1007/978-14614-8806-4 56

A. Dai, Z. Zhao, R. Li, H. Zhang and Y. Zhou, "Evaluation Mechanism of Collective Intelligence for Heterogeneous Agents Group," in IEEE Access, vol. 8, pp. 28385-28394, 2020, doi: 10.1109/ACCESS.2020.2971278.

R. Palak and N. T. Nguyen, "Prediction markets as a vital part of collective intelligence," 2017 IEEE International Conference on INnovations in Intelligent SysTems and Applications (INISTA), Gdynia, 2017, pp. 137-142, doi: 10.1109/INISTA.2017.8001146.

V. Nehra, R. Nagpal and R. Sehgal, "Collective Intelligence: When, Where and Why," 2020 10th International Conference on Cloud Computing, Data Science \& Engineering (Confluence), Noida, India, 2020, pp. 805-810, doi: 10.1109/Confluence47617.2020.9058000.

Peter Krafft, Kaitlyn Zhou, Isabelle Edwards, Kate Starbird, and Emma S Spiro. 2017. Centralized, parallel, and distributed information processing during collective sensemaking. In Proceedings of the 2017 CHI Conference on Human Factors in Computing Systems. ACM, 2976-2987.

Gimpel, Henner and Graf, Valerie and Hosseini, Sabiölla and Seyfried, Johannes, Analyzing the Emergence and Structure of Collective Intelligence in Human Groups - Pre-Registration of Study Design and Data Analysis Procedures (July 4, 2018). Available at SSRN:

https://ssrn.com/abstract $=3208325$

Toret, J., and Calleja, A. (2014) D2. 1 Collective intelligence framework, Universitat Oberta de Catalunya, accessed February 16th, 2016 at http://dcentproject.eu/wpcontent/uploads/2014/04/D2.1-.pdf

Maisha Joseph, William Moloney, Brian Cococcia, Kevin Goke, Tejasvini Shelar, Charles C. Tappert, Avery Leider, Collective Intelligence - A Group's Perspective, Proceedings of Student-Faculty Research Day, CSIS, Pace University, May 3th, 2019

Jordan B. Barlow, Alan R. Dennis, Collective intelligence' is not necessarily present in virtual groups, https://blogs.lse.ac.uk/businessreview/2017/01/11/collective-intelligence-is-not-necessarily-present-invirtual-groups/, January 11th, 2017, accessed Aug 19, 2020. 
Bernstein J, Long JS, Veillette C, Ahn J (2011) Crowd Intelligence for the Classification of Fractures and Beyond. PLoS ONE 6(11): e27620. https://doi.org/10.1371/journal.pone.0027620

Kudyba, S., Fjermestad, J. and Davenport, T. (2020), "A research model for identifying factors that drive effective decision-making and the future of work", Journal of Intellectual Capital, Vol. ahead-ofprint No. ahead-of-print. https://doi.org/10.1108/JIC-05-2019-0130

Morano, R.S., de Moraes, E.A. \& Jacomossi, R.R. Can small groups avoid the tragedy of the commons?. AI \& Soc 33, 71-80 (2018). https://doi.org/10.1007/s00146-017-0720-9

C W Callaghan, THE INTELLIGENCE OF THE CROWD: IMPLICATIONS FOR MANAGEMENT, PROCEEDINGS, 27th Annual SAIMS Conference 2015, page 1359, ISBN: 978-0-620-66504-9

Dr. Anna T. Mayo and Professor Anita Williams Woolley, 0: Variance in Group Ability to Transform Resources into Performance, and the Role of Coordinated Attention. AMD, 0 , https://doi.org/10.5465/amd.2019.0231

Callaghan, C. W. (2016). Knowledge management and problem solving in real time: The role of swarm intelligence. Interdisciplinary Journal of Information, Knowledge, and Management, 11, 177-199. Retrieved from http://www.informingscience.org/Publications/3528

Michelle P. Martin-Raugh, Patrick C. Kyllonen, Jiangang Hao, Adam Bacall, Dovid Becker, Christopher Kurzum, Zhitong Yang, Fred Yan, Patrick Barnwell, Negotiation as an interpersonal skill: Generalizability of negotiation outcomes and tactics across contexts at the individual and collective levels, Computers in Human Behavior, Volume 104, 2020, 105966, ISSN 0747-5632, https://doi.org/10.1016/j.chb.2019.03.030.

S. Sotiriadis, N. Bessis, E. Asimakopoulou and F. Xhafa, "Crowd-Sourcing and Data Mashups Challenges: A Mini Case Study for Assisting and Solving a Disaster Management Scenario," 2010 International Conference on Intelligent Networking and Collaborative Systems, Thessaloniki, 2010, pp. 496-501, doi: 10.1109/INCOS.2010.44.

Pease, A., Martin, U., Tanswell, F.S. et al. Using crowdsourced mathematics to understand mathematical practice. ZDM Mathematics Education (2020). https://doi.org/10.1007/s11858-020$\underline{01181-7}$

Malone T.W. (2020) How Can Human-Computer "Superminds" Develop Business Strategies? In: Canals J., Heukamp F. (eds) The Future of Management in an AI World. IESE Business Collection. Palgrave Macmillan, Cham. https://doi.org/10.1007/978-3-030-20680-2 9

Malone, Thomas, How Human-Computer 'Superminds' Are Redefining the Future of Work, W.MIT Sloan Management Review; Cambridge Vol. 59, Iss. 4, (Summer 2018): 34-41.

Cushman, F. and Gershman, S. (2019), Editors' Introduction: Computational Approaches to Social Cognition. Top Cogn Sci, 11: 281-298. doi:10.1111/tops.12424

Steinhart, E. Supermachines and Superminds. Minds and Machines 13, 155-186 (2003). https://doi.org/ $\underline{10.1023 / \mathrm{A}: 1021307923600}$ 
Laubacher, Robert and Giacomelli, Gianni and Kennedy, Kathleen and Kong, David Sun and Bachmann, Annalyn and Kramer, Katharina and Schlag, Paul and Malone, Thomas W., Using a Supermind to Design a Supermind: A Case Study of University Researchers and Corporate Executives Co-Designing an Innovative Healthcare Concept (March 31, 2020). MIT Center for Collective Intelligence, Massachusetts Institute of Technology, 2020, Available at SSRN:

https://ssrn.com/abstract=3601059 or http://dx.doi.org/10.2139/ssrn.3601059

Metcalf, L., Askay, D. A., \& Rosenberg, L. B. (2019). Keeping Humans in the Loop: Pooling Knowledge through Artificial Swarm Intelligence to Improve Business Decision Making. California Management Review, 61(4), 84-109. https://doi.org/10.1177/0008125619862256

Selmer Bringsjord, Michael John Zenzen, M. Zenzen, Superminds: People Harness Hypercomputation, and More, Volume 29 of Studies in Cognitive Systems, ISSN 0924-0780, Springer Science \& Business Media (2003), ISBN: 1402010958, 9781402010958

Andy E Williams. The Need for General Collective Intelligence in Addressing the Sustainable Development Goals, 19 March 2020, PREPRINT (Version 1) available at Research Square [+https://doi.org/10.21203/rs.3.rs-18022/v1+]

Williams, A. E. (2020, April 20). The Global Response to COVID-19 as an Example of a One-Sided Problem Definition in the Absence of General Collective Intelligence.

https://doi.org/10.31234/osf.io/emgxc

Williams, A. E. (2020, April 20). Transforming Economies and Generating Sustainable "Green" Economic Growth After the COVID-19 Pandemic through General Collective Intelligence. https://doi.org/10.31235/osf.io/arw7c 